\title{
Optimal Harvesting Policy of Predator-Prey Model with Free Fishing and Reserve Zones
}

\author{
Syamsuddin Toaha ${ }^{1, a)}$ and Rustam ${ }^{2, b)}$ \\ ${ }^{I}$ Department of Mathematics, Hasanuddin University, \\ Jln. Perintis Kemerdekaan, KM 10, 90245, \\ Makassar, Indonesia \\ ${ }^{2}$ Department of Mathematics, Universitas Sembilanbelas November, \\ Jln. Pemuda, Kolaka, Indonesia \\ a)syamsuddint@yahoo.com \\ b)rustam.math06@gmail.com
}

\begin{abstract}
The present paper deals with an optimal harvesting of predator-prey model in an ecosystem that consists of two zones, namely the free fishing and prohibited zones. The dynamics of prey population in the ecosystem can migrate from the free fishing to the prohibited zone and vice versa. The predator and prey populations in the free fishing zone are then harvested with constant efforts. The existence of the interior equilibrium point is analyzed and its stability is determined using Routh-Hurwitz stability test. The stable interior equilibrium point is then related to the problem of maximum profit and the problem of present value of net revenue. We follow the Pontryagin's maximal principle to get the optimal harvesting policy of the present value of the net revenue. From the analysis, we found a critical point of the efforts that makes maximum profit. There also exists certain conditions of the efforts that makes the present value of net revenue becomes maximal. In addition, the interior equilibrium point is locally asymptotically stable which means that the optimal harvesting is reached and the unharvested prey, harvested prey, and harvested predator populations remain sustainable. Numerical examples are given to verify the analytical results.
\end{abstract}

\section{INTRODUCTION}

The dynamics of predator and prey populations which consists of two populations or three populations is one of the topics of research in mathematical ecology which has been widely studied. Some authors considered two preys and one predator or one prey and two predators in the population dynamics. The other researchers studied two stages, mature and immature in the predator or in the prey, some others reseachers considered two zones of the ecosystem, namely a free fishing zone and the other is a prohibited zone where the predator or prey migrates between the two zones. In [1] the dynamics of a fishery resources in an aquatic ecosystem which consists of a free fishing zone and a prohibited zone was considered and the fish population was protected from over exploitation by controlling the tax. The system of predator-prey with two stages structure for predator and prey in a two parts of ecosystem with harvesting for mature predator and mature prey in the free fishing zone was anayzed by [2] and the analysis showed that there exists certain conditions to get the optimal harvesting.

The dynamics of predator and prey populations with selective harvesting has been deeply studied in many perspectives. Some authors considered harvesting for the prey population only (for examples in $[1,3,4,5]$ ), harvesting for the predator population only (for examples in $[6,7,8,9,10,11,12]$ ), and harvesting for both predator and prey populations (for examples in $[13,14]$ ). Most of predator-prey model with harvesting was related to the economic problems including maximum profit problem, taxation effect, and total discounted net revenue problem, for examples in $[1,14,15]$.

Based on the previous results of the authors, we investigate the dynamics of predator and prey populations with prey can migrate between the two zones, one of which is a free fishing zone and the other is a prohibited fishing 
zone. The predator and prey populations in the free fishing zone are then exploited with constant efforts. We analyze the existence and the stability of the interior equilibrium point. The stable interior equilibrium point is then related to the problem of maximum profit and to the problem of maximum present value of net revenue. We determine the critical point of the efforts that makes the profit function becomes maximum and also the present value of net revenue becomes maximum. The Routh-Hurwitz stability tes is applied to decide the kind of the stability of the interior equilibrium point. The Pontryagin's maximal principle is applied to find out the optimal harvesting policy of present value of net revenue.

\section{DYNAMICS OF PREDATOR AND PREY WITH PROHIBITED ZONE}

We consider a predator-prey fishery management in an ecosystem which consists of two zones, namely a free fishing zone and a prohibited zone, where no fishing is allowed in this zone. The two zones are supposed have the same characteristics, and the prey population can migrate in the two zones freely. The growth of the prey in the free fishing zone and in the prohibited zone in the absence of predator is assumed to be logistic. We assume that the predator just cathes the prey in the free fishing zone. The dynamics of predator and prey populations is denoted as a system of differential equations

$$
\begin{aligned}
& \frac{d x}{d t}=r x\left(1-\frac{x}{K}\right)-a_{1} x+a_{2} y-a_{3} x-\alpha x z \\
& \frac{d y}{d t}=s y\left(1-\frac{y}{L}\right)+a_{1} x-a_{2} y-a_{4} y \\
& \frac{d z}{d t}=m \alpha x z-k z .
\end{aligned}
$$

The symbols $x=x(t)$ and $y=y(t)$ state the size of prey population in the free fishing zone and in the prohibited zone at time $t$, respectively. The symbol $z=z(t)$ states the size of the predator population in the free fishing zone at time $t$. Parameter $r$ is the intrinsic growth rate of the prey population in the free fishing zone. Parameter $K$ is carrying capacity of the ecosystem for the prey population. The level of predation is expressed by the parameter $\alpha$, and the value of $m(0<m<1)$ is the scaled of predation to the predator. Parameter $s$ is the intrinsic growth of prey population in reserve zone, $L$ is the carrying capacity of the ecosystem for the prey population in the prohibited zone. Parameters $a_{3}, a_{4}$, and $k$ are the mortality rate for the prey in free fishing zone, prey in prohibited zone, and predator respectively. Parameter $a_{1}$ states the migration rate from the prey in the free fishing zone to the prey in prohibited zone, while parameter $a_{2}$ states the migration rate from the prey population in the prohibited zone to the prey population in the free fishing zone.

By considering that the populations are economically valuable, then the predator and the prey populations in free fishing zone are exploited with constant efforts. The dynamics of predator and prey populations is then extended and written in the form

$$
\begin{aligned}
& \frac{d x}{d t}=r x\left(1-\frac{x}{K}\right)-a_{1} x+a_{2} y-a_{3} x-\alpha x z-q_{1} E_{1} x \\
& \frac{d y}{d t}=s y\left(1-\frac{y}{L}\right)+a_{1} x-a_{2} y-a_{4} y \\
& \frac{d z}{d t}=m \alpha x z-k z-q_{3} E_{3} z .
\end{aligned}
$$

In the model (2), parameters $q_{1}$ and $q_{3}$ state the cathability coefficient for the prey and the predator populations respectively. Parameters $E_{1}$ and $E_{3}$ state the harvesting efforts satisfying $0 \leq E_{i} \leq E_{i \max }$ for $i=1,3$ and some value of $E_{i \max }$. Let $r_{1}=r / K, r_{2}=r-a_{1}-a_{3}, s_{1}=s / L, s_{2}=s-a_{2}-a_{4}$, and $a_{5}=m \alpha$, then model (2) becomes 


$$
\begin{aligned}
& \frac{d x}{d t}=r_{2} x-r_{1} x^{2}+a_{2} y-\alpha x z-q_{1} E_{1} x \\
& \frac{d y}{d t}=s_{2} y-s_{1} y^{2}+a_{1} x \\
& \frac{d z}{d t}=a_{5} x z-k z-q_{3} E_{3} z .
\end{aligned}
$$

From the model (3), we have three non negative equilibrium points, namely $T_{1}=(0,0,0), T_{2}=\left(x_{2}, y_{2}, 0\right)$,

and $\quad T_{3}=\left(x_{3}, y_{3}, z_{3}\right), \quad$ where $\quad x_{3}=\frac{q_{3} E_{3}+k}{a_{5}}, \quad y_{3}=\frac{s_{2}+\sqrt{s_{2}{ }^{2}+4 s_{1} a_{1} x_{3}}}{2 s_{1}}, \quad$ and $z_{3}=\frac{r_{2} x_{3}-r_{1} x_{3}{ }^{2}+a_{2} y_{3}-q_{1} E_{1} x_{3}}{\alpha x_{3}}$. When $r_{2} x_{3}-r_{1} x_{3}{ }^{2}+a_{2} y_{3}-q_{1} E_{1} x_{3}>0$, the equilibrium point $T_{3}$ becomes an interior equilibrium point. For analyzes we use the Jacobian matrix to get the linear model and by evaluating the Jacobian matrix at the equilibrium point $T_{3}=\left(x_{3}, y_{3}, z_{3}\right)$, we have

$$
J_{E}=\left(\begin{array}{ccc}
r_{2}-2 r_{1} x_{3}-\alpha z_{3}-q_{1} E_{1} & a_{2} & -\alpha x_{3} \\
a_{1} & s_{2}-2 s_{1} y_{3} & 0 \\
a_{5} z_{3} & 0 & a_{5} x_{3}-k-q_{3} E_{3}
\end{array}\right) .
$$

The polynomial characteristic associated with the Jacobian matrix $J_{E}$ is given by $f(\lambda)=\operatorname{det}\left(\lambda I-J_{E}\right)$, i.e

$$
\begin{aligned}
f(\lambda)=\lambda^{3}+ & b_{2} \lambda^{2}+b_{1} \lambda+b_{0}, \text { where } \\
b_{2}= & \alpha z_{1}+q_{3} E_{3}-s_{2}-r_{2}+q_{1} E_{1}+k-a_{5} x_{3}+2 s_{1} y_{3}+2 r_{1} x_{3}, \\
b_{1}= & -2 s_{1} y_{3} a_{5} x_{3}+2 s_{1} y_{3} q_{3} E_{3}+2 r_{1} x_{3} q_{3} E_{3}+\alpha z_{3} q_{3} E_{3}-q_{1} E_{1} a_{5} x_{3}+q_{1} E_{1} q_{3} E_{3}-2 r_{2} s_{1} y_{3} \\
& -2 r_{1} x_{3} s_{2}-\alpha z_{1} s_{2}-q_{1} E_{1} s_{2}-a_{1} a_{2}+r_{2} s_{2}+2 r_{1} x_{3} k+\alpha z_{1} k+r_{2} a_{5} x_{3}+q_{1} E_{1} k+2 s_{1} y_{1} k \\
& -r_{2} q_{3} E_{3}-2 r_{1} a_{5} x_{3}{ }^{2}-s_{2} q_{3} E_{3}+s_{2} a_{5} x_{3}-r_{2} k-s_{2} k+4 r_{1} x_{3} s_{1} y_{3}+2 \alpha z_{3} s_{1} y_{3}+2 q_{1} E_{1} s_{1} y_{3}, \\
b_{0}= & 4 r_{1} x_{3} s_{1} y_{3} q_{3} E_{3}+2 \alpha z_{3} s_{1} y_{3} q_{3} E_{3}-2 q_{1} E_{1} s_{1} y_{3} a_{5} x_{3}+2 q_{1} E_{1} s_{1} y_{3} q_{3} E_{3}+r_{2} s_{2} k-a_{1} a_{2} k \\
& +2 r_{2} s_{1} y_{3} a_{5} x_{3}-2 r_{2} s_{1} y_{3} q_{3} E_{3}-2 r_{1} s_{2} x_{3} q_{3} E_{3}-4 r_{1} s_{1} a_{5} y_{3} x_{3}{ }^{2}+4 r_{1} x_{3} s_{1} y_{3} k-\alpha z_{3} s_{2} q_{3} E_{3} \\
& +2 \alpha z_{3} s_{1} y_{3} k+q_{1} E_{1} s_{2} a_{5} x_{3}-q_{1} E_{1} s_{2} q_{3} E_{3}+2 q_{1} E_{1} s_{1} y_{3} k-r_{2} s_{2} a_{5} x_{3}+r_{2} s_{2} q_{3} E_{3}-2 r_{2} s_{1} y_{3} k \\
& +2 r_{1} s_{2} a_{5} x_{3}{ }^{2}-2 r_{1} x_{3} s_{2} k-\alpha z_{3} s_{2} k-q_{1} E_{1} s_{2} k+a_{1} a_{2} a_{5} x_{3}-a_{1} a_{2} q_{3} E_{3} .
\end{aligned}
$$

Referring to the Routh-Hurwitz criterion [16], the interior equilibrium point $T_{3}$ is locally asymptotically stable when the conditions $b_{0}>0, b_{2}>0$, and $b_{2} b_{1}-b_{0}>0$ are satisfied.

\section{BIOLOGICAL AND ECONOMIC EQUILIBRIUM}

The biological and economic equilibrium is a notion that integrates biological aspect and economical aspect in the population dynamics, as considered in [17]. The biological equilibrium means that there is not changes of the population, which is found by solving the equations $\frac{d x}{d t}=0, \frac{d y}{d t}=0$, and $\frac{d z}{d t}=0$ simultaneously. The economic equilibrium means that the total revenue from exploitation of populations equals to the total cost of exploitation efforts. We just consider that the total cost is proportional to the exploitation effort, written as $T C=C(E)=c E$. We also suppose that the total revenue from the exploitation of population is proportional to the exploitation yield, written as $T R=p Y(E)$, where $p$ is the unit price of the stock and $Y(E)=E q x$ denotes the exploitation yield function which has been studied in [18]. The profit function is then given by $\pi=T R-T C$. Let $c_{i}$ states exploitation cost per unit effort of population $x_{i}$ and $p_{i}$ denoted the price per unit biomass of population $x_{i}$. The profit function of exploitation for predator and prey populations in the free fishing zone is given by 
$\pi=\left(p_{1} q_{1} x\right) E_{1}+\left(p_{3} q_{3} z\right) E_{3}-\left(c_{1} E_{1}+c_{3} E_{3}\right)$. The bionomic equilibrium $\left(x^{*}, y^{*}, z^{*}, E_{1}^{*}, E_{3}^{*}\right)$ is found by solving the following simultaneous equation

$$
\begin{aligned}
& r_{2} x-r_{1} x^{2}+a_{2} y-\alpha x z-q_{1} E_{1} x=0 \\
& s_{2} y-s_{1} y^{2}+a_{1} x=0 \\
& a_{5} x z-k z-q_{3} E_{3} z=0 \\
& \left(p_{1} q_{1} x\right) E_{1}+\left(p_{3} q_{3} z\right) E_{3}-\left(c_{1} E_{1}+c_{3} E_{3}\right)=0 .
\end{aligned}
$$

We then associate the interior equilibrium point $T_{3}$ to the maximum profit problem. The point $T_{3}$ is an interior equilibrium point if the conditions $0 \leq E_{i} \leq E_{i \max }$ for $i=1,3$ and

are satisfied.

$$
r_{2} x_{3}-r_{1} x_{3}^{2}+a_{2} y_{3}-q_{1} E_{1} x_{3}>0
$$

The profit function at the equilibrium point $T_{3}$ is written in the form

$$
\pi\left(E_{1}, E_{3}\right)=\left(p_{1} q_{1} x_{3}\right) E_{1}+\left(p_{3} q_{3} z_{3}\right) E_{3}-\left(c_{1} E_{1}+c_{3} E_{3}\right) .
$$

The problem is determining a pair of efforts $\left(E_{1}, E_{3}\right)$ which satisfies (4) and $0 \leq E_{i} \leq E_{i \max }$ for $i=1,3$ that maximizes the profit function $\pi\left(E_{1}, E_{3}\right)$. We also need the interior equilibrium point $T_{3}$ is always asymptotically stable.

\section{OPTIMAL HARVESTING POLICY OF THE POPULATION}

The biological equilibrium is found by evaluating the equations $\frac{d x}{d t}=0, \frac{d y}{d t}=0$, and $\frac{d z}{d t}=0$ simultaneously.

The economic equilibrium is found when the total revenue equals the total cost. The profit function for the exploited predator and prey populations is written as $\pi\left(E_{1}, E_{3}\right)=p_{1} q_{1} x E_{1}+p_{3} q_{3} z E_{3}-c_{1} E_{1}-c_{3} E_{3}$. Our goal is to maximize the present value $J$ of net revenue function which is given by

$$
J=\int_{0}^{\infty} e^{-\delta t}\left\{\left(p_{1} q_{1} x-c_{1}\right) E_{1}(t)+\left(p_{3} q_{3} z-c_{3}\right) E_{3}(t)\right\} d t
$$

The symbol $\delta$ states the discount rate of the net revenue. Our goal is to maximize the present value $J$ subject to the equation (3) by following the Pontryagin's maximum principle [19]. Variables $E_{1}(t)$ and $E_{3}(t)$ as a control are subject to the condition $0 \leq E_{i}(t) \leq E_{i \max }$ for $i=1,3$.

From this problem we write the Hamiltonian function as

$$
\begin{aligned}
H= & e^{-\delta t}\left\{\left(p_{1} q_{1} x-c_{1}\right) E_{1}+\left(p_{3} q_{3} z-c_{3}\right) E_{3}\right\}+\lambda_{1}\left\{r_{2} x-r_{1} x^{2}+a_{2} y-\alpha x z-q_{1} E_{1} x\right\} \\
& +\lambda_{2}\left\{s_{2} y-s_{1} y^{2}+a_{1} x\right\}+\lambda_{3}\left\{a_{5} x z-k z-q_{3} E_{3} z_{3}\right\},
\end{aligned}
$$

where the variables $\lambda_{1}(t), \lambda_{2}(t)$, and $\lambda_{3}(t)$ state the adjoints of the problem.

We set $\frac{\partial H}{\partial E_{1}}=0$ and $\frac{\partial H}{\partial E_{3}}=0$ as the necessary conditions for the control variables $E_{1}$ and $E_{3}$ to be optimal. From the Hamiltonian function (6), we have $\frac{\partial H}{\partial E_{1}}=e^{-\delta t}\left(p_{1} q_{1} x-c_{1}\right)-\lambda_{1} q_{1} x=0$ and $\frac{\partial H}{\partial E_{3}}=e^{-\delta t}\left(p_{3} q_{3} z-c_{3}\right)-\lambda_{3} q_{3} z=0$. Then we get $\lambda_{1}=\frac{e^{-\delta t}\left(p_{1} q_{1} x-c_{1}\right)}{q_{1} x}$ and $\lambda_{3}=\frac{e^{-\delta t}\left(p_{3} q_{3} z-c_{3}\right)}{q_{3} z}$. From the Hamiltonian equation we also have

$$
\begin{aligned}
& \frac{\partial H}{\partial x}=e^{-\delta t} p_{1} q_{1} E_{1}+\lambda_{1}\left(r_{2}-2 r_{1} x-\alpha z-q_{1} E_{1}\right)+\lambda_{2} a_{1}+\lambda_{3} a_{5} z \\
& \frac{\partial H}{\partial y}=+\lambda_{1} a_{2}+\lambda_{2}\left(s_{2}-2 s_{1} y\right), \text { and }
\end{aligned}
$$




$$
\frac{\partial H}{\partial z}=e^{-\delta t} p_{3} q_{3} E_{3}-\lambda_{1} \alpha x+\lambda_{3}\left(a_{5} x-k-q_{3} E_{3}\right) .
$$

From the Pontryagin's maximum principle $\dot{\lambda}_{1}=-\frac{\partial H}{\partial x}, \dot{\lambda}_{2}=-\frac{\partial H}{\partial y}$, and $\dot{\lambda}_{3}=-\frac{\partial H}{\partial z}$ we get

$$
\begin{aligned}
& \frac{\delta e^{-\delta t}\left(-p_{1} q_{1} x+c_{1}\right)}{q_{1} x}+e^{-\delta t} p_{1} q_{1} E_{1}+\lambda_{1}\left(r_{2}-2 r_{1} x-\alpha z-q_{1} E_{1}\right)+\lambda_{2} a_{1}+\lambda_{3} a_{5} z=0, \\
& \dot{\lambda}_{2}+\left(s_{2}-2 s_{1} y\right) \lambda_{2}+a_{2} \lambda_{1}=0, \text { and } \\
& \frac{\delta e^{-\delta t}\left(-p_{3} q_{3} z+c_{3}\right)}{q_{3} z}+e^{-\delta t} p_{3} q_{3} E_{3}-\lambda_{1} \alpha x+\lambda_{3}\left(a_{5} x-k-q_{3} E_{3}\right)=0 .
\end{aligned}
$$

By substituting $\lambda_{1}=\frac{e^{-\delta t}\left(p_{1} q_{1} x-c_{1}\right)}{q_{1} x}$ into (8) and considering transversality condition $\lambda_{2}(t)=0$ as $t \rightarrow \infty$, we get $\quad \lambda_{2}=\frac{e^{-\delta t} a_{2}\left(-p_{1} q_{1} x+c_{1}\right)}{q_{1} x\left(-\delta+s_{2}-2 s_{1} y\right)}$. Again, substituting $\quad \lambda_{1}=\frac{e^{-\delta t}\left(p_{1} q_{1} x-c_{1}\right)}{q_{1} x}, \quad \lambda_{2}=\frac{e^{-\delta t} a_{2}\left(-p_{1} q_{1} x+c_{1}\right)}{q_{1} x\left(-\delta+s_{2}-2 s_{1} y\right)}, \quad$ and $\lambda_{3}=\frac{e^{-\delta t}\left(p_{3} q_{3} z-c_{3}\right)}{q_{3} z}$ into equations (7) and (9) we get $E_{1}$ and $E_{3}$, where

$$
\begin{aligned}
E_{1} & =\frac{1}{q_{1} q_{3} c_{1}\left(\delta-s_{2}+2 s_{1} y\right)}\left\{2 \alpha p_{1} q_{1} q_{3} s_{1} x y z-\delta p_{1} q_{1} q_{3} s_{2} x-\delta r_{2} p_{1} q_{1} q_{3} x+r_{2} p_{1} q_{1} q_{3} s_{2} x+2 \delta r_{1} p_{1} q_{1} q_{3} x^{2}\right. \\
& -2 r_{1} s_{2} p_{1} q_{1} q_{3} x^{2}-4 r_{1} q_{3} c_{1} s_{1} x y-2 \alpha c_{1} s_{1} q_{3} y z-a_{1} a_{2} p_{1} q_{1} q_{3} x+2 a_{5} q_{1} c_{3} s_{1} x y+p_{1} q_{1} q_{3} x \delta^{2}-2 \delta c_{1} q_{3} s_{1} y \\
& +2 r_{2} q_{3} c_{1} s_{1} y-2 x r_{1} q_{3} c_{1} \delta+2 x r_{1} q_{3} c_{1} s_{2}-\alpha q_{3} z c_{1} \delta+\alpha q_{3} z c_{1} s_{2}+a_{5} x q_{1} c_{3} \delta-a_{5} x q_{1} c_{3} s_{2}+\delta c_{1} q_{3} s_{2} \\
& +r_{2} q_{3} c_{1} \delta-r_{2} q_{3} c_{1} s_{2}-2 a_{5} x q_{1} p_{3} q_{3} s_{1} y^{2}+2 \delta p_{1} q_{1} x q_{3} s_{1} y+\alpha q_{3} z p_{1} q_{1} x \delta+4 r_{1} q_{3} p_{1} q_{1} s_{1} y x^{2} \\
& \left.-2 r_{2} q_{3} p_{1} q_{1} s_{1} x y-\alpha q_{3} z p_{1} q_{1} x s_{2}+a_{1} a_{2} q_{3} c_{1}+a_{5} x q_{1} p_{3} q_{3} y s_{2}-a_{5} x q_{1} p_{3} q_{3} y \delta-c_{1} q_{3} \delta^{2}\right\}, \\
E_{3} & =\frac{1}{q_{1} q_{3}\left(p_{3} q_{3} z-p_{3} q_{3} y+c_{3}\right)}\left\{\delta p_{3} q_{1} q_{3} z-\delta c_{3} q_{1}+a_{5} q_{1} c_{3} x+\alpha p_{1} q_{1} q_{3} x z-\alpha c_{1} q_{3} z\right. \\
& \left.-a_{5} p_{3} q_{1} q_{3} x y+k p_{3} q_{1} q_{3} y-k q_{1} c 3\right\} .
\end{aligned}
$$

By substituting $x=x_{3}=\frac{q_{3} E_{3}+k}{a_{5}}, y=y_{3}=\frac{s_{2}+\sqrt{s_{2}{ }^{2}+4 s_{1} a_{1} x_{3}}}{2 s_{1}}$, and $z=z_{3}=\frac{r_{2} x_{3}-r_{1} x_{3}{ }^{2}+a_{2} y_{3}-q_{1} E_{1} x_{3}}{\alpha x_{3}}$ into equations (10) and (11) we get the value of control variables $E_{1}$ and $E_{3}$. The values of $E_{1}, E_{3}, x_{3}, y_{3}$, and $z_{3}$ maximize the present value of $J$.

\section{NUMERICAL EXAMPLES}

Based on the analytical results above, we give some examples to simulate the problem of maximum profit and the problem of maximizing the present value of net revenue.

For the problem of maximum profit function, we set the parameter values as $r=1.5, a_{1}=0.0018$, $a_{2}=0.0175, \quad a_{3}=0.0001, a_{4}=0.0001 \quad K=100000, \quad \alpha=0.15, \quad s=1.4, \quad L=110000, \quad m=0.112$, $k=0.0001, q_{1}=4.1$, and $q_{3}=4.5$ in appropriate units. Take $p_{1}=25, p_{3}=15, c_{1}=1000$, and $c_{3}=1020$ in appropriate units. Then we have the equilibrium point $T_{3}=\left(x_{3}, y_{3}, z_{3}\right)$, where

$$
\begin{aligned}
& x_{3}=0.0059524+267.85714 E_{3}, \\
& y_{3}=54308.571+39285.714 \sqrt{1.9110298+0.0000245 E_{3}}, \text { and }
\end{aligned}
$$




$$
\begin{aligned}
z_{3} & =\frac{1}{0.0008929+40.178571 E_{3}}\left\{-0.0001500\left(0.0059524+267.85714 E_{3}\right)^{2}\right. \\
& +950.40892+401.27679 E_{3}+687.49999 \sqrt{1.9110298+0.0000245 E_{3}} \\
& \left.-4.1 E_{1}\left(0.0059524+267.85714 E_{3}\right)\right\} .
\end{aligned}
$$

In order for equilibrium point $T_{3}$ to be an interior equilibrium point, the exploitation efforts $E_{1}$ and $E_{3}$ must satisfy the conditions $P_{3}>0$ where

$$
\begin{aligned}
P_{3} & =\left\{-0.0001500\left(0.0059524+267.85714 E_{3}\right)^{2}+950.40892+401.27679 E_{3}\right. \\
& \left.+687.49999 \sqrt{1.9110298+0.0000245 E_{3}}-4.1 E_{1}\left(0.0059524+267.85714 E_{3}\right)\right\}
\end{aligned}
$$

and $0 \leq E_{i}(t) \leq E_{i \max }$ for $i=1,3$. We let $E_{1 \max }=1$ and $E_{3 \max }=1$. In the other words, the equilibrium point $T_{3}$ becomes an interior point when $\left(E_{1}, E_{3}\right) \in D_{1}$, where $D_{1}=\left\{\left(E_{1}, E_{3}\right): 0 \leq E_{1} \leq 1,0 \leq E_{3} \leq 1\right\}$.

The profit function associates with the equilibrium point $T_{3}$ is given by

$\pi\left(E_{1}, E_{3}\right)=\left(p_{1} q_{1} x_{3}\right) E_{1}+\left(p_{3} q_{3} z\right) E_{3}-\left(c_{1} E_{1}+c_{3} E_{3}\right)$. After substituting the values of $x_{3}, y_{3}, z_{3}$ and simplifying we get

$$
\begin{aligned}
\pi\left(E_{1}, E_{3}\right)= & \left\{-999.38988+27455.357 E_{3}\right\} E_{1}+\left\{63132.602+27086.183 E_{3}\right. \\
& -0.0010125\left(0.0059524+267.85714 E_{3}\right)^{2}+46406.249 \sqrt{1.9110298+0.0000245 E_{3}} \\
& \left.-276.75 E_{1}\left(0.0059524+267.85714 E_{3}\right)\right\} E_{3} .
\end{aligned}
$$

We have a stationary point $\left(E_{1}^{*}, E_{3}^{*}\right)=(0.0116921,0.0390229)$ in $D_{1}$ and the three critical points $\left(E_{1}^{*}, E_{3}^{*}\right)=(0,0),\left(E_{1}^{*}, E_{3}^{*}\right)=(0,1)$, and $\left(E_{1}^{*}, E_{3}^{*}\right)=(1,1)$ at the boundary of $D_{1}$. Unfortunately, the critical point $\left(E_{1}^{*}, E_{3}^{*}\right)=(0.0116921,0.0390229)$ which gives $\pi\left(E_{1}^{*}, E_{3}^{*}\right)=3178.0279$ is not a maximum value. The critical point $\left(E_{1}^{*}, E_{3}^{*}\right)=(0,1)$ maximizes the profit function with the value of $\pi(0,1)=154298.55$. In this case, there is not harvesting for the prey in the free fishing zone while the predator is harvested at maximum level of effort. By applying the value of harvesting efforts $\left(E_{1}^{*}, E_{3}^{*}\right)=(0,1)$ we get the equilibrium point $T_{3}=(267.86309,1086174.9,57.268448)$. The polynomial characteristic of the Jacobian matrix is given by $f(\lambda)=\lambda^{3}+8.4826119 \lambda^{2}+48.472413 \lambda+53.439865$ which has the eigenvalues -1.3824103 and $-3.5501008 \pm 5.1042929 i$. Under this condition, if we take the values of efforts $E_{1}^{*}=0$ and $E_{3}^{*}=1$, then the predator and the prey populations in the two zones will sustain for a long period of time and also maximize the profit function.

For the problem of maximizing present value of the net revenue, we set the parameter values as $r_{1}=10.5$, $a_{1}=0.180, a_{2}=0.175, a_{3}=0.0001, a_{4}=0.0001, K=100000, \alpha=0.0045, m=0.0212, s=10.4, L=110000$, $k=0.0001, q_{1}=5.1$, and $q_{3}=9.5$ in appropriate units. Take $p_{1}=10, p_{3}=10, c_{1}=1000, c_{3}=1050$, and $\delta=0.003$ in appropriate units. Then we have the optimal interior equilibrium point $T_{3}=\left(x_{3}, y_{3}, z_{3}\right)$, where

$$
\begin{aligned}
x_{3} & =1.0482180+99580.713 E_{3}, \\
y_{3} & =54073.990+5.5434607 \sqrt{9.5151400 \times 10^{-7}+6.1694313 \times 10^{-8} E_{3}}, \text { and } \\
z_{3}= & \frac{222.22222}{1.0482180+99580.713 E_{3}}\left\{-0.0001050\left(1.0482180+99580.713 E_{3}\right)^{2}\right. \\
& +9473.7658+1027662.9 E_{3}+9701056.3 \sqrt{9.5151400 \times 10^{-7}+6.1694313 \times 10^{-8} E_{3}} \\
& \left.-5.1 E_{1}\left(1.0482180+99580.713 E_{3}\right)\right\} .
\end{aligned}
$$

The adjoint variables are 


$$
\begin{aligned}
& \lambda_{1}=\frac{-0.1960784 e^{-0.003 t}\left(946.54088-5078616.3 E_{3}\right)}{1.0482180+99580.713 E_{3}}, \\
& \lambda_{2}=\frac{0.0343137 e^{-0.003 t}\left(946.54088-5078616.3 E_{3}\right)}{\left(1.0482180+99580.713 E_{3}\right)\left(-10482.180 \sqrt{9.5151400 \times 10^{-7}+6.1694313 \times 10^{-8} E_{3}}\right)}, \text { and } \\
& \lambda_{3}=\frac{-4.74 \cdot 10^{-11} e^{-0.003 t}\left(1.28 \cdot 10^{15}+7.89 \cdot 10^{9} \sqrt{2.64 \cdot 10^{10}+1.71 \cdot 10^{9} E_{3}}\right)\left(1.05 \cdot 10^{9}+9.96 \cdot 10^{13} E 3\right)}{-2.4 \cdot 10^{14}-2.6 \cdot 10^{16} E_{3}+2.6 \cdot 10^{16} E_{3}^{2}-1.5 \cdot 10^{9} \sqrt{2.6 \cdot 10^{10}+1.7 \cdot 10^{9} E_{3}}+1.3 \cdot 10^{11} E_{1}+1.3 \cdot 10^{16} E_{1} E_{3}} .
\end{aligned}
$$

After substituting and solving the related equations we get the harvesting efforts $E_{1}=0.0221549$ and $E_{3}=0.9947320$. Then we get the interior equilibrium point $T_{3}=(99057.168,109864.55,0.0000728)$. The eigenvalues associted wit the interior equilibirum point are $-10.751232,-10.393349$, and $-2.945142 \cdot 10^{-7}$. Under this condition, the interior equilibrium point $T_{3}$ is locally asymptotically stable. The adjoint variables are written as $\lambda_{1}=9.9980206 e^{-0.003 t}, \quad \lambda_{2}=0.1658049 e^{-0.003 t}$, and $\lambda_{3}=1.5099596 \cdot 10^{10} e^{-0.003 t}$. Then, we get the maximum value of present value of the net revenue $J=\int_{0}^{\infty} 1.1085806 \cdot 10^{5} e^{-0.003 t} d t=3.6952688 \cdot 10^{7}$.

\section{CONCLUSIONS}

The dynamics of predator and prey populations with constant harvesting efforts for the predator and the prey populations in the free fishing zone is possible to have an interior equilibrium point $T_{3}=\left(x_{3}, y_{3}, z_{3}\right)$. The interior equilibrium point exists when the values of paramaters and harvesting efforts satisfy the conditions $r_{2} x_{3}-r_{1} x_{3}{ }^{2}+a_{2} y_{3}-q_{1} E_{1} x_{3}>0$ and $0 \leq E_{i} \leq E_{i \max }$. The interior equilibrium point $T_{3}$ is locally asymptotically stable when the certain conditions are satisfied. The existence and the stability of the interior equilibrium point $T_{3}$ depends on the values of parameters and harvesting efforts. With the condition $0 \leq E_{i} \leq E_{i \max }$, there exists a situation such that the interior equilibrium point $T_{3}$ is always locally asymptotically stable and also maximizes the profit function. The predator and the prey populations can live in coexistence for a long period of time, although the predator and the prey populations in the free fishing zone are exploited with constant efforts. In addition, the harvested populations in the free fishing zone also give maximum profit. By following the Pontryagin's maximum principle, we found that there exists a specific value of harvesting efforts for the predator and the prey populations in free fishing zone that maximizes the present value of the net revenue. Beside that, the predator and the prey populations in the two zones will not be extinct.

\section{REFERENCES}

1. H.F. Hou, H.M. Jiang and X.Y. Meng. A dynamic model for fishery resource with reserve area and taxation. Journal of Applied Mathematics, (2012). http://dx.doi.org/10.1155/2012/794719.

2. M.N. Srinivas, M.A.S. Srinivas, K. Das and N.H. Gazi. Prey-predator fishery model with stage structure in two patchy marine aquatic environment. Applied Mathematics 2, 1405-1416 (2011).

3. K. Chakraborty, M. Chakraborty and T.K. Kar. Bifurcation and control of a bioeconomic model of a preypredator system with time delay. Nonlinear Analysis: Hybrid Systems 5, 613-625 (2011).

4. R.P. Gupta, M. Banerjee and P. Chandra. Bifurcation analysis and control of Leslie-Gower predator-prey model with Michaelis-Menten type prey-harvesting. Journal Differential Equations and Dynamical Systems 22(3), 339-366 (2012).

5. T.K. Kar. A Dynamic reaction model of a prey-predator system with stage-structure for predator. Modern Applied Science 4(5), 183-195 (2010).

6. G.M.V. Laksmi, M. Gunasekaran and S. Vijaya. Bifurcation analysis of predator model with harvested predator. International Journal of Engineering Research and Development 10(6), $42-51$ (2014). 
7. C. Liu, Q. Zhang and J. Li. Global stability analysis and optimal control of a harvested ecoepidemiological prey predator model with vaccination and taxation. Abstract and Applied Analysis. Article ID 950396, 16 pages. (2013). http://dx.doi.org/10.1155/2013/950396.

8. K. Chakraborty, M Chakraborty and T.K. Kar. Optimal control and bifurcation of a prey-predtor model with stage structure. Applied Mathematics and Computation 217(21), 8778-8792 (2011).

9. Y. Qu and J. Wei. Bifurcation analysis in a predator-prey system with stage stucture and harvesting. Journal of Franklin Institute 347(7), 1096-1113 (2010).

10. K. Chakraborty, S. Jana and T.K. Kar. Global dynamics and bifurcation in a stage structured prey-predator fishery model with harvesting. Applied Mathematics and Computation 218(5), 9271-9290 (2012).

11. C. Liu and W. Tang. The dynamics and control of a harvested differential-algebraic prey-predator model. International Journal of Information and System Sciences 7(1), 103-113 (2011).

12. C. Liu, Q. Zhang and X. Duan. Dynamical behavior in a harvested differential-algebraic prey-predator model with discrete time delay and stage structure. Journal of the Franklin Institute 346(10), 1038-1059 (2009).

13. K. Chakraborty, M. Chakraborty and T.K. Kar. Optimal control of harvest and bifurcation of a prey-predator model with stage structure. Applied Mathematics and Computation 217, 8778-8792 (2011).

14. S. Toaha, J. Kusuma, Khaeruddin and Mawardi. Stabilty analysis and optimal harvesting policy of preypredator model with stage structure for predator. Applied Mathematical Sciences 8(159), 7923-7934 (2014).

15. K. Chakraborty, M. Chakraborty and T.K. Kar. Optimal control of harvest and bifurcation of prey-predator model with stage structure. Applied Mathematics and Computation 217, 8778-8792 (2011).

16. L.E. Keshet, Mathematical models in biology. SIAM, Philadelphia, 2005, pp. 231-234

17. D.K. Bhattacharya and S. Begum. Bionomic equilibrium of two-species system, Mathematical Biosciences 135, 111-127 (1996).

18. C.W. Clark, Bioeconomic modelling and fisheries management. John Wiley \& Sons, New York, 1985, pp. 1115

19. D. Grass, J.P. Caulkins, G. Feichtinger, G. Tragler and D.A. Behrens, Optimal control of nonlinear processes. Springer-Verlag, Berlin Heidelberg, 2003, pp. 122-138. 